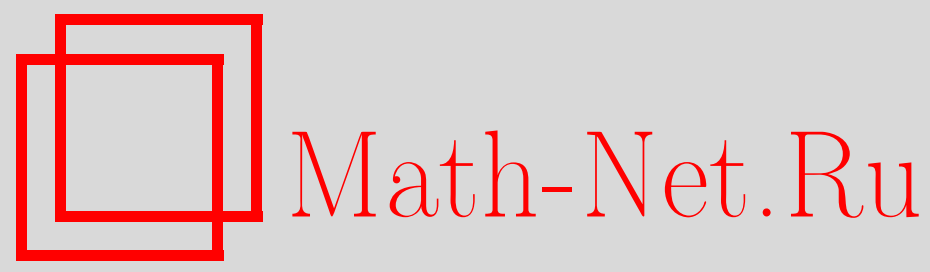

А. М. Чеботарев, Г. В. Рыжаков, О сильной резольвентной сходимости шредингеровской эволюции к квантовой стохастике, Матем. заметки, 2003, том 74, выпуск 5, 762-781

DOI: https://doi.org/10.4213/mzm297

Использование Общероссийского математического портала Math-Net.Ru подразумевает, что вы прочитали и согласны с пользовательским соглашением http://www.mathnet.ru/rus/agreement

Параметры загрузки:

IP : 54.198 .55 .26

26 апреля 2023 г., 06:58:38

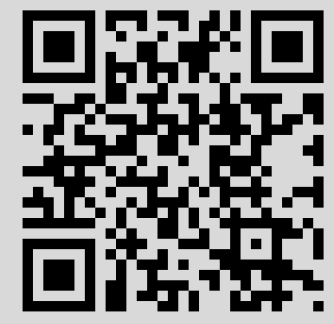




\title{
О СИЛЬНОЙ РЕЗОЛЬВЕНТНОЙ СХОДИМОСТИ ШРЕДИНГЕРОВСКОЙ ЭВОЛЮЦИИ К КВАНТОВОЙ СТОХАСТИКЕ
}

\author{
А. М. Чеботарев, Г.В. Рыжаков
}

\begin{abstract}
В статье доказана сильная сходимость шредингеровской эволюции к квантовой стохастике для класса гамильтонианов, включщего модель квантового детектора гравитационньх волн. Доказано, что сильньп резольвентным пределом последовательности самосопряженных гамильтонианов является симметричная краевая задача в фоковском пространстве и что для предельной эволюции частичного следа по смешанному состоянию не существует единого уравнения типа уравнения Линдблада. Напротив, каждая компонента смешанного состояния в пределе порождает собственный закон эволюции.
\end{abstract}

Библиографияя: 15 названий.

1. Введение. Начиная с экспериментов Резерфорда по рассеянию $\alpha$-частиц на тонкой золотой фольге, в квантовых измерениях используются макроскопические пучки квантовых частищ, взаимодействующие с микроскопическими квантовьми системами таким образом, что параметры пучка, доступные для измерения с помощю классических приборов до и после рассеяния, содержат информацию о состоянии квантовой системы. Одним из примеров макроскопического квантового пучка, используемого в интерферометрических измерениях микроскопических смещений, является пучок когерентных фотонов, создаваемьй лазером. Длина оптического пути фотонов намного больше области взаимодействия с квантовой системой, поэтому такое взаимодействие может быть описано как изменение фазы и амплитуды исходного пучка, происходящее в пределе в одной точке, лежащей на его оптическом пути.

В качестве примера мы рассмотрим модель квантового детектора гравитационных волн. Он состоит из источника когерентного излучения и оптической интерферометрической системы. Осциллятор взаимодействует с падающей гравитационной волной и его смещения создают фазовые сдвиги волн когерентного излучения, наблюдаемые как изменения интенсивности сигнала от фотодетектора. Математическая модель измерительного прибора выглядит следующим образом.

Пусть $\Gamma^{S}=\Gamma^{S}\left(L_{2}(\mathbb{R})\right)$ - симметричное фоковское пространство со скалярным произведением

$$
(\phi, \psi)=\bar{\phi}_{0} \psi_{0}+\sum_{n=1}^{\infty} \int_{\mathbb{R}^{n}} d x \bar{\phi}_{n}(x) \psi_{n}(x), \quad \phi_{0}, \psi_{0} \in \mathbb{C}, \quad \phi_{n}, \psi_{n} \in L_{2}\left(\mathbb{R}^{n}\right) .
$$




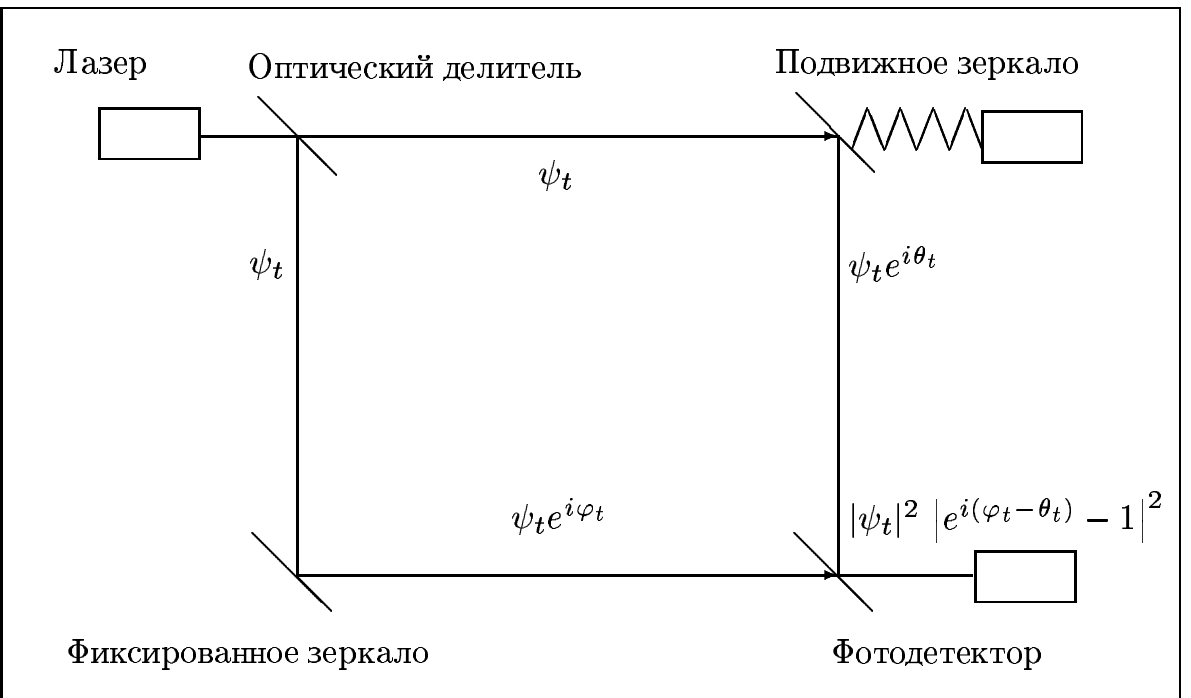

Рис. 1. Фрагмент интерферометра типа LIGO

Пространство $\Gamma^{S}$ является гильбертовым пространством состояний пучка фотонов.

Амплитуда вероятности числа фотонов в луче, распространяющемся вдоль оптического пути со скоростью $c$, описьвается нормированным симметричным фоковским вектором $\psi_{t} \in \Gamma^{S}\left(L_{2}(\mathbb{R})\right)$ :

$$
\psi_{t}=\left\{\psi_{0}(t), \psi_{1}\left(x_{1}+c t\right), \psi_{2}\left(x_{1}+c t, x_{2}+c t\right), \ldots\right\},
$$

а координаты $x$ вдоль оптической оси выбраны таким образом, чтобы зеркало в невозмущенном состоянии находилось в начале координат. С точностью до нормировки функция $P_{N}(x, t)=\left|\psi_{N}(x+c t)\right|^{2}$ является плотностью вероятности в $N$-частичном подпространстве. Так как функции $\psi_{N}(x)$ симметричны относительно перестановок компонент вектора $x=\left\{x_{1}, \ldots, x_{N}\right\}$, вероятность $P_{n}(B, t)$ обнаружить ровно $n$ частищ на множестве $B \subseteq \mathbb{R}$ вычисляется по формуле

$$
\begin{aligned}
P_{n}(B, t) & =\sum_{N=n}^{\infty} C_{N}^{n} \int_{B^{n}} \int_{(\mathbb{R} \backslash B)^{N-n}} d x P_{N}(x, t) \\
& =\left.\frac{1}{n !} \frac{d^{n}}{d \lambda^{n}} \sum_{N=0}^{\infty} \int_{\mathbb{R}^{N}} d x P_{N}(x, t) \prod_{k=1}^{N}\left(\lambda I_{B}\left(x_{k}\right)+I_{\mathbb{R} \backslash B}\left(x_{k}\right)\right)\right|_{\lambda=0} .
\end{aligned}
$$


Поэтому среднее значение числа частиц на множестве $B$ в момент времени $t$ равно

$$
\begin{aligned}
N(B, t) & =\sum_{n=0}^{\infty} n P_{n}(B, t)=\left.\frac{d}{d \lambda} e^{\frac{d}{d \lambda}} \sum_{N=0}^{\infty} \int_{\mathbb{R}^{N}} d x P_{N}(x, t) \prod_{k=1}^{N}\left(\lambda I_{B}\left(x_{k}\right)+I_{\mathbb{R} \backslash B}\left(x_{k}\right)\right)\right|_{\lambda=0} \\
& =\left.\frac{d}{d \lambda} \sum_{N=0}^{\infty} \int_{\mathbb{R}^{N}} d x P_{N}(x, t) \prod_{k=1}^{N}\left(1+\lambda I_{B}\left(x_{k}\right)\right)\right|_{\lambda=0} \\
& =\sum_{N=0}^{\infty} \int_{\mathbb{R}^{N}} d x\left|\psi_{N}(x+c t)\right|^{2} \sum_{k=1}^{N} I_{B}\left(x_{k}\right)=\left(\psi_{t}, \int_{\mathbb{R}} I_{B}(x) a^{\dagger}(x) a(x) d x \Psi_{t}\right)_{\Gamma^{S}},
\end{aligned}
$$

где $I_{B}(x)$ - индикаторная функция множества $B$. Последнее равенство следует из определения операторов рождения и уничтожения в фоковском пространстве $G^{S}$. Таким образом, оператор

$$
\widehat{N}(B)=\int_{\mathbb{R}} I_{B}(x) a^{\dagger}(x) a(x) d x, \quad \widehat{N}(B) \psi_{N}(x)=\sum_{k=1}^{N} I_{B}\left(x_{k}\right) \psi_{N}(x), \quad x=\left(x_{1}, \ldots, x_{N}\right)
$$

интерпретируется как оператор числа частиц на множестве $B$.

Пространством состояний квантового осциллятора является гильбертово пространство $\ell_{2}$, а гамильтониан свободного осциллятора равен $\hbar \Omega b^{\dagger} b$, где $\Omega$ - собственная частота осциллятора, $a b^{\dagger}, b$ - соответственно операторы рождения и уничтожения, действующие в $\ell_{2}$. Свободная эволюция пучка фотонов и осциллятора описывается уравнением Шрёдингера

$$
\frac{\hbar}{i} \frac{\partial}{\partial t} \Psi_{t}=\left\{-i \hbar c \nabla+\hbar \Omega b^{\dagger} b\right\} \Psi_{t}
$$

где $\hbar$ - постоянная Планка, и $(\nabla \psi)_{n}\left(x_{1}, \ldots, x_{n}\right)=\sum_{k} \frac{\partial}{\partial x_{k}} \psi_{n}\left(x_{1}, \ldots, x_{n}\right)$. Чтобы описать взаимодействие излучения с осциллятором, рассмотрим силу, создаваемую в точке $x$ потоком падающих в единицу времени $N(x)$ фотонов на зеркало, перемещающееся вдоль оптической оси. Она равна импульсу $p=N(x) \hbar \omega / c$, передаваемому фотонами зеркалу за единицу времени, где $\omega$ - частота лазерного излучения, $x-$ координата осциллятора. Таким образом, классическая и квантованная потенциальная энергия зеркала равны соответственно

$$
E(x)=\frac{\hbar \omega}{c} \int_{0}^{x} d y N(y), \quad \widehat{E}=\frac{\hbar \omega}{c} \int_{0}^{g \frac{b^{\dagger}+b}{\sqrt{2}}} d y a^{\dagger}(y) a(y)
$$

где $\widehat{x}=g \frac{b^{\dagger}+b}{\sqrt{2}}-$ каноническое квантование координаты $x$ осциллятора, $g$ - постоянная. Если на осциллятор действует внешняя сила $f(t)$, то полный оператор энергии запишется в следуюшем виде:

$$
\widehat{H}=\hbar \Omega b^{\dagger} b-i c \hbar \nabla+\frac{\hbar \omega}{c} \int_{0}^{g \frac{b^{\dagger}+b}{\sqrt{2}}} a^{\dagger}(x) a(x) d x+g \frac{b^{\dagger}+b}{\sqrt{2}} f(t)
$$


Оператор $\widehat{x}=g \frac{b^{\dagger}+b}{\sqrt{2}}$ является самосопряженным, поэтому гамильтониан (1.1) может быть корректно выражен через интеграл по спектральному семейству оператора $\widehat{x}$.

Решение задачи Коши для уравнения Шрёдингера с гамильтонианом (1.1) неизвестно, а приближенное, которое может быть построено аналитически, является нетривиальньп. Такое приближение оправдано, если плотность числа фотонов мало меняется на интервалах, сравнимых с амплитудой колебаний осциллятора. В этом случае с целью регуляризации сингулярных возмущений рассмотрим последовательность функций $\left\{g_{\alpha}(x)\right\}_{\alpha \in(0,1]}$ такую, что при $\alpha \rightarrow 0$ существует слабьй предел $g_{\alpha}(x) \rightarrow \delta(x)$ и

$$
g \frac{b^{\dagger}+b}{\sqrt{2}} \int_{\mathbb{R}} g_{\alpha}(x) a^{\dagger}(x) a(x) d x \underset{\alpha \rightarrow 0}{\longrightarrow} g \frac{b^{\dagger}+b}{\sqrt{2}} a^{\dagger}(0) a(0) \approx \int_{0}^{g \frac{b^{\dagger}+b}{\sqrt{2}}} a^{\dagger}(x) a(x) d x .
$$

Формально гамильтониан (1.1) может быть аппроксимирован сингулярно возмущенным оператором

$$
\widehat{H}_{s}=\Omega \hbar b^{\dagger} b-i c \hbar \nabla+g \frac{b^{\dagger}+b}{\sqrt{2}}\left(\hbar \omega a^{\dagger}(0) a(0)+f(t)\right),
$$

главная часть которого $g \frac{b^{\dagger}+b}{\sqrt{2}} a^{\dagger}(0) a(0)$ равна нулю на плотном подмножестве, состоящем из линейной оболочки элементов $\Psi(v) \otimes h$, где

$$
\Psi(v)=\left\{1, \frac{v\left(x_{1}\right)}{\sqrt{1 !}}, \frac{v\left(x_{1}\right) v\left(x_{2}\right)}{\sqrt{2 !}}, \ldots\right\} \in \Gamma^{S}\left(L_{2}(\mathbb{R})\right)
$$

- экспоненщиальный вектор, $v \in C_{0}^{\infty}(\mathbb{R}) \subset L_{2}(\mathbb{R}), v(0)=0, h \in\left(I+b^{\dagger} b\right)^{-1} \ell_{2}$. Поэтому гамильтониан (1.2) является сингулярно возмущенным оператором [1]. Как будет показано ниже, сильньй резольвентный предел последовательности самосопряженных операторов

$$
\widehat{H}=\hbar \Omega b^{\dagger} b-i c \hbar \nabla+\hbar \omega g \frac{b^{\dagger}+b}{\sqrt{2}} \int_{\mathbb{R}} g_{\alpha}(x) a^{\dagger}(x) a(x) d x+g \frac{b^{\dagger}+b}{\sqrt{2}} f(t)
$$

отличается от (1.2); он равен замыканию оператора

$$
C=\Omega \hbar b^{\dagger} b-i c \hbar \nabla+g \frac{b^{\dagger}+b}{\sqrt{2}} f
$$

заданного на области определения, состоящей из функций, принадлежащих $\mathscr{D}_{W}=$ $\Gamma^{S}\left(W_{2}^{1}(\mathbb{R} \backslash\{0\}) \otimes\left(I+b^{\dagger} b\right)^{-1} l_{2}\right.$ и удовлетворяющих краевому условию

$$
A_{-} \Psi=W A_{+} \Psi, \quad W=e^{i g \omega \frac{b^{\dagger}+b}{\sqrt{2}}}
$$

где $A_{ \pm} \Psi(v) \stackrel{\text { def }}{=} v( \pm 0) \Psi(v), \forall v \in W_{2}^{1}(\mathbb{R} \backslash\{0\})$. Множество $\mathscr{D}_{W}$ содержит линейную оболочку векторов $\psi\left(\hat{v}_{\varepsilon}\right) \otimes h, h \in\left(I+b^{\dagger} b\right)^{-1} l_{2}$,

$$
\hat{v}_{\varepsilon}(x)=v(x)\left(1-\xi\left(\frac{x}{\varepsilon}\right)\right)+c \xi\left(\frac{x}{\varepsilon}\right)\left(I_{(-\infty, 0)}(x) W+I_{(0, \infty)}(x)\right),
$$


где $v \in L_{2}(\mathbb{R}), \quad \xi \in C_{0}^{\infty}(\mathbb{R}), \quad \xi(x)=1$ при $|x| \leqslant 1, \varepsilon \in(0,1), c \in \mathbb{C}$. Поскольку $\hat{v}_{\varepsilon}(-0)=W \hat{v}_{\varepsilon}(+0)$, очевидно, что эти функции принадлежат $\mathscr{D}_{W}$ и удовлетворяют краевому условию (1.4) и $\psi\left(\hat{v}_{\varepsilon}\right) \rightarrow \psi(v)$ при $\varepsilon \rightarrow 0$. Поскольку множество экспоненциальных векторов тотально в $\Gamma^{S}$, a $\left(I+b^{\dagger} b\right)^{-1} l_{2}$ плотно в $\ell_{2}$, то множество $\mathscr{D}_{W}$ плотно в $\mathfrak{H}=\Gamma^{S} \otimes l_{2}$.

В разделе 2 показано, что существует унитарная группа $U_{t}=e^{i t \widehat{C}}$, порождаемая самосопряженным оператором $\widehat{C}=C^{* *}$, унитарно эквивалентна разрешаюшему оператору квантового стохастического дифференциального уравнения

$$
d u_{t}=u_{t}\left((W-I) \Lambda(d t)+\left(i \Omega b^{\dagger} b+i g \frac{b^{\dagger}+b}{\hbar \sqrt{2}} f(t)\right) d t\right), \quad u_{t}=e^{-c t \nabla} U_{t},
$$

с процессом числа частиц

$$
\Lambda(T)=\int_{T} d t a^{\dagger}(c t) a(c t)=\left.\int_{T} d t e^{c t \nabla} a^{\dagger}(x) a(x) e^{-c t \nabla}\right|_{x=0}
$$

Среднее значение этого оператора совпадает со средним числом фотонов, взаимодействуюших с зеркалом за время $T$. Уравнения $(1.3),(1.4)$ дают правильную формулу квантования сдвига фазы, вытекающую из известной формулы светового давления. В общем случае оператор $W$ можно рассматривать как оператор рассеяния макроскопического тока на квантовой системе.

2. Сильная резольвентная сходимость в фоковском пространстве: гамильтонианы с коммутирующими операторными коэффициентами. Пусть $\mathscr{H}$ гильбертово пространство, $\Gamma^{S}\left(L_{2}(\mathbb{R})\right)$ - симметричное фоковское пространство и $\mathfrak{H}=$ $\mathscr{H} \otimes \Gamma^{S}\left(L_{2}(\mathbb{R})\right)$ - их тензорное произведение. Для $g \in L_{2}(\mathbb{R}) \cap L_{1}(\mathbb{R}) \cap C(\mathbb{R})$ через

$$
A^{\dagger}(g) \stackrel{\text { def }}{=} \int d x g(x) a^{\dagger}(x) \quad \text { и } \quad A(g) \stackrel{\text { def }}{=} \int d x g(x) a(x)
$$

обозначим стандартные операторы рождения и уничтожения, действующие в $\Gamma^{S}\left(L_{2}(\mathbb{R})\right), \Lambda(g) \stackrel{\text { def }}{=} \int d x g(x) a^{\dagger}(x) a(x)$. Рассмотрим уравнение Шрёдингера $\partial \psi_{t} / \partial t=$ $i \mathbf{H} \psi_{t}$ с гамильтонианом

$$
\mathbf{H}=H_{0} \otimes I-i \otimes \nabla+H_{\mathrm{int}}, \quad H_{\mathrm{int}}=K \otimes \Lambda(g)+R \otimes A^{\dagger}(g)+R^{*} \otimes A(g),
$$

имеюшим некоторую плотную область определения $\mathscr{D}_{\mathbf{H}} \subset \mathfrak{H}$. Для простоты в этом разделе предполагается, что операторы $H_{0}, K, R$ принадлежат множеству замкнутых операторов $\mathscr{C}(\mathscr{H})$ и попарно коммутируют, т.е. имеют общее спектральное семейство $E_{\lambda}$ и общую плотную область определения $\mathscr{D} \subset \mathscr{H}$. Более точно, пусть

$$
H_{0}=\int \nu_{\lambda} d E_{\lambda}, \quad K=\int \lambda d E_{\lambda}, \quad R=\int \rho_{\lambda} e^{i \Phi_{\lambda}} d E_{\lambda}, \quad \lambda \in \mathbb{R},
$$

где $\nu_{\lambda}, \rho_{\lambda}$ и $\Phi_{\lambda}$ - измеримые вещественные функции, соответствующие операторам $H_{0}$, $K$ и $R$ такие, что операторы $H_{0}$ и $K$ являются самосопряженными, а $R$ - нормальным. 
Обозначим через $\widehat{P}_{t}^{\lambda}=e^{i \hat{\delta} t}$ однопараметрическую групу унитарных операторов в $L_{2}(\mathbb{R})$ с генератором $\hat{\delta}=-i \partial_{x}+\lambda g(x)$, т.е.

$$
\widehat{P}_{t}^{\lambda} f(x)=f(x+t) e^{i \lambda \int_{0}^{t} d s g(x+t-s)} .
$$

Унитарная група $U_{t}=\exp \{i \mathbf{H} t\}$ может быть построена явно путем преобразования гамильтониана (2.1) к канонической форме или переходом к представлению взаимодействия, порождаемому оператором $-I \otimes i \nabla+K \otimes \Lambda(g)$. Следующее утверждение описьвает действие $U_{t}$ на когерентные векторы $\psi(v) \in \Gamma^{S}\left(L_{2}(\mathbb{R})\right), v \in L_{2}(\mathbb{R})$, при этом $U_{t} \psi(v)$ является оператором, действующим в гильбертовом пространстве $\mathscr{H}:\left(U_{t} \psi(v)\right) h=$ $U_{t}(\psi(v) \otimes h)$.

ТЕОРема 2.1. Унитарная однопараметрическая группа $U_{t}=\exp \{i t \mathbf{H}\}$ действует по правилу

$$
U_{t} \psi(v)=\int d E_{\lambda} \psi\left(v_{t}^{\lambda}\right) \exp \left\{i \nu_{\lambda} t+i \rho_{\lambda} e^{-i \Phi_{\lambda}} \int_{0}^{t}\left(g, v_{s}^{\lambda}\right) d s\right\},
$$

əде

$$
\begin{aligned}
v_{t}^{\lambda}(x) & =\widehat{P}_{t}^{\lambda} v(x)+i \rho_{\lambda} e^{i \Phi_{\lambda}} \int_{0}^{t} \widehat{P}_{t-s}^{\lambda} g(x) d s \\
& =e^{i \lambda \int_{0}^{t} d s g(x+t-s)} v(x+t)+i \rho_{\lambda} e^{i \Phi_{\lambda}} \int_{0}^{t} g(x+t-s) e^{i \lambda \int_{0}^{s} d \tau g(x+s-\tau)} d s .
\end{aligned}
$$

ДоКАЗАТЕЛЬСТВО. Прямьми вычислениями нетрудно установить групповое свойство, слабую непрерьвность и унитарность семейства $U_{t}$. Благодаря групповому свойству сильная непрерьвность следует из слабой, а генератор групш достаточно вычислить при $t=0$ в слабом смысле, используя правило дифференцирования экспоненциальных векторов:

$$
\left(\psi(w), \frac{d}{d t} \psi\left(v_{t}\right)\right)_{\Gamma^{S}}=\left(\psi(w), A^{\dagger}\left(v_{t}^{\prime}\right) \psi\left(v_{t}\right)\right)_{\Gamma^{S}}=\left(\psi(w), \psi\left(v_{t}\right)\right)_{\Gamma^{S}} \frac{d}{d t}\left(w, v_{t}\right)_{L_{2}}
$$

По определению семейства $v_{t}^{\lambda}(x)$ имеем

$$
\begin{aligned}
\left(w, v_{t}^{\lambda}\right)= & \int_{\mathbb{R}} d x \overline{w(x)}\left(e^{i \lambda \int_{0}^{t} d s g(x+t-s)} v(x+t)\right. \\
& \left.+i \rho_{\lambda} e^{i \Phi_{\lambda}} \int_{0}^{t} d \tau e^{i \lambda \int_{0}^{t-\tau} d s g(x+t-\tau-s)} g(x+t-\tau)\right) .
\end{aligned}
$$

Простые вычисления показьвают, что генератор равен $i \mathbf{H : ~ ч л е н ы ~} i H_{0}+i R^{*} \otimes A(g)$ получаются при дифференцировании экспоненты в $(2.2)$, а члены $\nabla+i K \otimes \Lambda(g)+R \otimes A^{\dagger}(g)$ возникают при дифференцировании $\psi\left(v_{t}\right)$ согласно приведенной вьше формуле. В унитарно-эквивалентном представлении такие вычисления подробно проделаны в [2], [3].

Пусть $g \in L_{2}(\mathbb{R}) \cap L_{1}(\mathbb{R}) \cap C(\mathbb{R})$ - положительная функиия с единичной $L_{1}$-нормой, и пусть $g_{\alpha}(x)=\alpha^{-1} g\left(\alpha^{-1} x\right)$; нетрудно видеть, что $g_{\alpha}(x) \rightarrow \delta(x)$ слабо при $\alpha \rightarrow 0$ на множестве непрерывных ограниченных функций $C(\mathbb{R})$. 
Лемма 2.1. Пусть $\widehat{P}_{t}^{(\alpha, \lambda)}$ - однопараметрическая унитарная әруппа в $L_{2}(\mathbb{R}) c$ генератором $-i \nabla+\lambda g_{\alpha}(x)$, и пусть $I_{T}-$ проектор, который действует в $L_{2}(\mathbb{R})$ как оператор умножения на индикаторную функиию $I_{T}(x)$ борелевского множества Т. Тогда при $\alpha \rightarrow 0$ существуют следующие пределы:

1) $\lim \int_{0}^{t} d s\left(g_{\alpha}, \widehat{P}_{s}^{(\alpha, \lambda)} g_{\alpha}\right)=\left\{\frac{e^{i \lambda}-1-i \lambda}{(i \lambda)^{2}}, t>0 ; \frac{1-i \lambda-e^{-i \lambda}}{(i \lambda)^{2}}, t<0\right\}$

2) $w-\lim \int_{0}^{t} d s \widehat{P}_{s}^{(\alpha, \lambda)} g_{\alpha}(x)=$ $=\left\{\frac{e^{i \lambda}-1}{i \lambda} I_{(-t, 0)}(x), t>0 ;-\frac{e^{-i \lambda}-1}{i \lambda} I_{(t, 0)}(x), t<0\right\}$

3) $\lim \int_{0}^{t} d s\left(g_{\alpha}, \widehat{P}_{s}^{(\alpha, \lambda)} v\right)=$ $=\left\{\frac{e^{i \lambda}-1}{i \lambda} \int_{0}^{t} d x v(x), t>0 ; \quad \frac{1-e^{-i \lambda}}{i \lambda} \int_{t}^{0} d x v(x), t<0\right\}$

4) $u-\lim \widehat{P}_{t}^{(\alpha, \lambda)} v(x)=\left\{e^{i \lambda I_{(-t, 0)}(x)} v(x+t), t>0 ; e^{-i \lambda I_{(0,-t)}(x)} v(x+t), t<0\right\}$.

ДокАЗАТЕЛЬСтво. Левые части формул 1)-4) записаны в обозначениях, соответствующих случаю $t>0$. В случае $t<0$ пределы интегрирования и аргументы индикаторных функций изменяются в соответствии с принятыми правилами: нижний предел интегрирования меньше верхнего, левый конец интервала меньше правого.

Для краткости мы рассмотрим случай $t>0$, поскольку в случае $t<0$ все формулы вьводятся аналогично. Докажем первое утверждение леммы. Из определения $g_{\alpha}$ следует

$$
\begin{aligned}
\int_{0}^{t} d s\left(g_{\alpha}, \widehat{P}_{s}^{(\alpha, \lambda)} g_{\alpha}\right) & =\frac{1}{\alpha^{2}} \int_{\mathbb{R}} d x g\left(\frac{x}{\alpha}\right) \int_{0}^{t} d s g\left(\frac{x+s}{\alpha}\right) e^{\frac{i \lambda}{\alpha} \int_{0}^{s} d \tau g\left(\frac{x+\tau}{\alpha}\right)} \\
& =\int_{\mathbb{R}} d x \int_{0}^{t / \alpha} d s g(x) g(x+s) e^{i \lambda \int_{0}^{s} d \tau g(x+\tau)} .
\end{aligned}
$$

Так как $g \in L_{1}(\mathbb{R}) \cap C(\mathbb{R})$, последний интеграл ограничен абсолютно интегрируемой функцией на $\mathbb{R}^{2}$ :

$$
\left|g(x) g(x+s) I_{(0, t / \alpha)}(s) e^{i \lambda \int_{0}^{s} d \tau g(x+\tau)}\right| \leqslant g(x) g(x+s)
$$

при любом $\alpha$. Таким образом, можно применить теорему Лебега о предельном переходе под знаком интеграла: последний интеграл сходится к явно вычисляемому повторному интегралу

$$
\int_{\mathbb{R}} d x g(x) \int_{0}^{\infty} d s g(x+s) e^{i \lambda \int_{0}^{s} d \tau g(x+\tau)}=\left(e^{i \lambda}-1-i \lambda\right)(i \lambda)^{-2},
$$

поскольку по предположению $\int_{\mathbb{R}} g(x) d x=\|g\|_{L_{1}}=1$. 
Доказательство второго утверждения следует из формулы интегрирования по частям:

$$
\begin{aligned}
\frac{1}{\alpha} \int_{0}^{t} d s g & \left(\frac{x+s}{\alpha}\right) e^{\frac{i \lambda}{\alpha} \int_{0}^{s} d \tau g\left(\frac{x+\tau}{\alpha}\right)}=\frac{1}{i \lambda} \int_{0}^{t} d e^{\frac{i \lambda}{\alpha} \int_{0}^{s} d \tau g\left(\frac{x+\tau}{\alpha}\right)} \\
= & \frac{1}{i \lambda}\left(e^{\frac{i \lambda}{\alpha} \int_{0}^{t} d \tau g\left(\frac{x+\tau}{\alpha}\right)}-1\right) \rightarrow \frac{1}{i \lambda}\left(e^{i \lambda I_{(-t, 0)}(x)}-1\right)=\frac{1}{i \lambda}\left(e^{i \lambda}-1\right) I_{(-t, 0)}(x) .
\end{aligned}
$$

Чтобы доказать третье соотношение, запишем скалярное произведение следуюшим образом:

$$
\begin{aligned}
\int_{0}^{t} d s\left(g_{\alpha}, \widehat{P}_{s}^{(\alpha, \lambda)} v\right) & =\int_{\mathbb{R}} d x v(x) \int_{0}^{t} d s \frac{1}{\alpha} g\left(\frac{x-s}{\alpha}\right) e^{\frac{i \lambda}{\alpha} \int_{0}^{s} d \tau g\left(\frac{x-\tau}{\alpha}\right)} \\
& =\frac{1}{i \lambda} \int_{\mathbb{R}} d x v(x) \int_{0}^{t} d e^{\frac{i \lambda}{\alpha} \int_{0}^{s} d \tau g\left(\frac{x-\tau}{\alpha}\right)} \\
& =\frac{1}{i \lambda} \int_{\mathbb{R}} d x v(x)\left(e^{\frac{i \lambda}{\alpha} \int_{0}^{t} d \tau g\left(\frac{x-\tau}{\alpha}\right)}-1\right) \\
& \rightarrow \frac{1}{i \lambda} \int_{\mathbb{R}} d x\left(e^{i \lambda I_{(0, t)}(x)}-1\right) v(x)=\frac{1}{i \lambda}\left(e^{i \lambda}-1\right) \int_{0}^{t} d x v(x) .
\end{aligned}
$$

Доказательство последнего соотношения следует из сильной сходимости последовательности унитарных полугруш $\widehat{P}_{t}^{(\alpha, \lambda)}$ к $e^{i \lambda I_{(-t, 0)}(x)} e^{t \nabla}$ при $\alpha \rightarrow+0$. Лемма доказана.

Лемма 2.1 позволяет перейти к пределу в формуле (2.2) при $\alpha \rightarrow+0$. Пределы 1$)-4)$ соответствуют переходу к пределу в четырех компонентах решения $(2.2)$, зависяших от $\alpha$. Подставляя 1)-4) в (2.2), получаем предельное семейство операторов $U_{t}=\exp \{i \mathbf{H} t\}=$ $s-\lim _{\alpha \rightarrow 0} \exp \left\{i \mathbf{H}_{\alpha} t\right\}$ при $t>0$ :

$$
U_{t} \psi(v)=\int d E_{\lambda} \exp \left\{\left(i H_{\lambda}-h_{\lambda}\right) t+\rho_{\lambda} e^{-i \Phi_{\lambda}} \frac{1}{\lambda}\left(e^{i \lambda}-1\right) \int_{0}^{t} v(x) d x\right\} \psi\left(v_{t}^{\lambda}\right),
$$

где $H_{\lambda}=\nu_{\lambda}+\rho_{\lambda}^{2}(\sin \lambda-\lambda) / \lambda^{2} \leqslant \nu_{\lambda}, h_{\lambda}=\rho_{\lambda}^{2}(1-\cos \lambda) / \lambda^{2} \geqslant 0$, и $v_{t}^{\lambda}(x)=e^{i \lambda I_{(-t, 0)}(x)} \times$ $v(x+t)+\rho_{\lambda} e^{i \Phi_{\lambda}} I_{(-t, 0)}(x)\left(e^{i \lambda}-1\right) / \lambda, e^{i \lambda I_{(-t, 0)}(x)}=\left(e^{i \lambda}-I\right) I_{(-t, 0)}(x)+I$. Положим $W_{\lambda} \stackrel{\text { def }}{=} e^{i \lambda}$ и $L_{\lambda} \stackrel{\text { def }}{=} \rho_{\lambda} e^{i \Phi_{\lambda}}\left(e^{i \lambda}-1\right) / \lambda$; эти функции являются спектральными плотностями операторов $W=e^{i K}$ и $L=\left(e^{i K}-I\right) K^{-1} R,-L^{*} W=R^{*}\left(e^{i K}-I\right) K^{-1}$. В таких обозначениях функция $\rho_{\lambda} e^{-i \Phi_{\lambda}} \frac{1}{\lambda}\left(e^{i \lambda}-1\right)$ соответствует оператору $R^{*} K^{-1}(W-$ $I)=-L^{*} W, h_{\lambda}$ соответствует оператору $L^{*} L / 2$, а $H_{\lambda}$ соответствует оператору

$$
H=H_{0}-R^{*} \frac{K-\sin K}{K^{2}} R
$$

Поэтому действие предела унитарных груш $U_{t}^{(\alpha)}$ может быть записано следующим образом:

$$
\begin{aligned}
U_{t} \psi(v)= & \psi\left(\left(W I_{(-t, 0)}(x)+I_{\mathbb{R} \backslash(-t, 0)}(x)\right) v(x+t)\right. \\
& \left.+L I_{(-t, 0)}(x)\right) e^{\left(i H-L^{*} L / 2\right) t-L^{*} W \int_{0}^{t} v(s) d s}
\end{aligned}
$$


При $t<0$ получаем предельное семейство операторов, действующих по правилу

$$
\begin{aligned}
U_{t} \psi(v)= & \psi\left(\left(W^{*} I_{(0,-t)}(x)+I_{\mathbb{R} \backslash(0,-t)}(x)\right) v(x+t)\right. \\
& \left.-W^{*} L I_{(0,-t)}(x)\right) e^{\left(i H+L^{*} L / 2\right) t+L^{*} \int_{t}^{0} v(s) d s} .
\end{aligned}
$$

Прямыми вычислениями на тотальном множестве когерентных векторов проверяется, что $U_{-t} U_{t}=I$ для любых $t \in \mathbb{R}$ и $\left(U_{t} \psi(w) \otimes g, U_{t} \psi(v) \otimes h\right)_{\mathfrak{H}}=(\psi(w), \psi(v))_{\Gamma}(g, h)_{\mathscr{H}}$ для всех $v, w \in L_{2}(\mathbb{R}), g, h \in \mathscr{H}$, поэтому семейство операторов $U_{t}$ является унитарным. Груповые свойства семейства операторов $U_{t}$ следуют из явного представления $(2.4),(2.5)$.

Для каждого фиксированного $t$ лемма 2.1 обеспечивает слабую сходимость $U_{t}^{(\alpha)} \rightarrow$ $U_{t}$ при $\alpha \rightarrow+0$. Так как нормы групп $U_{t}^{(\alpha)}$ и $U_{t}$ равны единице, из слабой сходимости следует сильная [4]. Таким образом, семейство гамильтонианов

$$
\mathbf{H}_{\alpha}=H_{0} \otimes I-I \otimes i \nabla+K \otimes \Lambda\left(g_{\alpha}\right)+R \otimes A^{\dagger}\left(g_{\alpha}\right)+R^{*} \otimes A\left(g_{\alpha}\right),
$$

сходится в смысле сильной резольвентной сходимости к некоторому самосопряженному оператору $\mathbf{H}$, являющемуся генератором унитарной группы $U_{t}[4]$, однако операторная реализация этого генератора не очевидна.

Найдем предельньй генератор Н. Для этого введем два сингулярных оператора уничтожения, которые действуют на экспоненциальных векторах следующим образом:

$$
A_{ \pm} \psi(v) \stackrel{\text { def }}{=} v( \pm 0) \psi(v), \quad v \in W_{2}^{1}(\mathbb{R} \backslash\{0\}) .
$$

Формулы (2.4), (2.5) показывают, что аргумент $\hat{v}_{t}$ экспоненциального вектора $\psi$ действует как операторозначная функция от $x \in \mathbb{R}$ в $\mathscr{H}$, причем при любом $t \in \mathbb{R}$ эта функция испытывает скачок в точках $x=-t$ и $x=0$, даже если $v(x)$ - гладкая функция. При $t>0$ из (2.4) имеем

$$
A_{+} U_{t} \psi(v)=v(t) U_{t} \psi(v), \quad A_{-} U_{t} \psi(v)=(W v(t)+L) U_{t} \psi(v), \quad W=e^{i K} .
$$

При $t<0$ аналогичное соотношение получаем из (2.5):

$$
A_{+} U_{t} \psi(v)=W^{*}(v(t)-L) U_{t} \psi(v), \quad A_{-} U_{t} \psi(v)=v(t) U_{t} \psi(v)
$$

Таким образом, для всех $t \in \mathbb{R}$ векторы из области значений унитарной группы $U_{t}$ удовлетворяют граничному условию

$$
\left(A_{-}-W A_{+}-L\right) \psi=0 \text {. }
$$

В случае ограниченных операторозначных коэффициентов плотным в $\mathfrak{H}$ подмножеством, удовлетворяющим краевому условию (2.7), является линейная оболочка векторов вида $\psi\left(\hat{v}_{\varepsilon}\right) \otimes h$, где

$$
\hat{v}_{\varepsilon}(x)=v(x)\left(1-\xi\left(\frac{x}{\varepsilon}\right)\right)+\xi\left(\frac{x}{\varepsilon}\right)\left(I_{(-\infty, 0)}(x)(W c+L)+c I_{(0, \infty)}(x)\right), \quad \varepsilon \in(0,1),
$$


$v \in W_{2}^{1}(\mathbb{R}), h \in \mathscr{H}, c \in \mathbb{C}, \xi \in C_{0}^{\infty}(\mathbb{R}), \xi(x)=1$ при $|x|<1$. Это условие описьвает операторозначные скачки фазы и амплитуды поля при отражении фотонов от зеркала в точке $x=0$, где происходит сингулярное взаимодействие между электромагнитным полем и квантовой системой. Дифференцируя явное представление групшы и учитывая, что $(\partial / \partial t+\partial / \partial x) I_{(-t, 0)}(x)=0$ при $x \neq 0$, получаем

$$
\begin{array}{ll}
-i \frac{d}{d t} U_{t} \psi(v)=\left(H+\frac{i L^{*} L}{2}-i \nabla+i L^{*} W A_{+}\right) U_{t} \psi(v), & t>0 \\
-i \frac{d}{d t} U_{t} \psi(v)=\left(H-\frac{i L^{*} L}{2}-i \nabla+i L^{*} A_{-}\right) U_{t} \psi(v), & t<0
\end{array}
$$

для $\psi \in \Gamma^{S}\left(W_{2}^{1}(\mathbb{R} \backslash\{0\})\right) \otimes \mathscr{D}$. Нетрудно видеть, что с учетом граничного условия $(2.7)$ правые части формул (2.8) совпадают. Таким образом, доказано следующее утверждение, описьвающее весьма нетривиальную связь операторных коэффициентов семейства гамильтонианов (2.6) и сильного резольвентного предела этой последовательности.

ТЕОРема 2.2. Сильным резольвентным пределом семейства операторов

$$
\mathbf{H}_{\alpha}=H_{0}-i \nabla+K \Lambda\left(g_{\alpha}\right)+R A^{\dagger}\left(g_{\alpha}\right)+R^{*} A\left(g_{\alpha}\right), \quad g_{\alpha}(x) \rightarrow \delta(x),
$$

является оператор $\mathbf{H}=H+i L^{*} L / 2-i \nabla+i L^{*} W A_{+}=H-i L^{*} L / 2-i \nabla+i L^{*} A_{-}$,

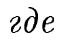

$$
H=H_{0}-R^{*}(K-\sin K) K^{-2} R, \quad W=e^{i K}, \quad L=-i(W-I) K^{-1} R,
$$

заданный на подмножестве векторов из $\Gamma^{S}\left(W_{2}^{1}(\mathbb{R} \backslash\{0\})\right) \otimes \mathscr{D}$, удовлетворяющих однородному граничному условию $\left(A_{-}-W A_{+}-L\right) \psi=0$.

Как было показано в [2] в случае ограниченных операторных коэффициентов (не обязательно коммутирующих), граничное условие (2.6) обеспечивает симметричность оператора Н. Область определения операторов Н с неограниченными операторными коэффициентами $H_{0}, K, R$ описана в [5]. Важно также отметить, что сильньй резольвентный предел семейства операторов (2.5) зависит от способа слабой аппроксимации сингулярного возмущения $a^{\dagger}(0) a(0)$. Если вместо использованной вьше физически мотивированной аппроксимации $\int_{\mathbb{R}} g_{\alpha}(x) a^{\dagger}(x) a(x) d x$ рассмотреть последовательность

$$
\int_{\mathbb{R}} g_{\alpha}(x) a^{\dagger}(x) d x \int_{\mathbb{R}} g_{\alpha}(y) a(y) d y
$$

имеющую тот же слабьй предел, то, как показано в [2], в утверждении леммы 2.1 и теоремы 2.2 изменится выражение для унитарного оператора $W$; в этом случае он равен $W=(K-2 i)(K+2 i)^{-1}$.

Закончим раздел вьводом квантового стохастического дифференциального уравнения и квантового уравнения Лиувилля (quantum master equation или master Markov equation), связанного с унитарной группой $U_{t}$. Сначала рассмотрим унитарный коцикл 
$u(t, s) \stackrel{\text { def }}{=} e^{-t \nabla} U_{t-s} e^{s \nabla}$, и найдем слабое дифференциальное уравнение для семейства $u_{t}=u(t, 0)$. Заметим, что $e^{-t \nabla} I_{(-t, 0)}(x)=I_{(0, t)}(x)$ при $t>0$; таким образом,

$$
\begin{aligned}
& u_{t} \psi(v)=\psi\left(\hat{v}_{t}\right) e^{\left(i H-L^{*} L / 2\right) t-L^{*} W \int_{0}^{t} v(s) d s}, \\
& \hat{v}_{t}=\left((W-I) I_{(0, t)}(x)+I\right) v(x)+L I_{(0, t)}(x) .
\end{aligned}
$$

Так как для производной экспоненциального вектора $\psi\left(\hat{v}_{t}\right)$ вьполнены соотношения

$\frac{d}{d t}\left(\psi(w), \psi\left(\hat{v}_{t}\right)\right)_{\Gamma^{S}}=\left(w, \hat{v}_{t}^{\prime}\right)_{L_{2}}\left(\psi(w), \psi\left(\hat{v}_{t}\right)\right)_{\Gamma^{S}}, \quad\left(w, \hat{v}_{t}^{\prime}\right)_{L_{2}}=(W-I) \bar{w}(t) v(t)+L \bar{w}(t)$

а дифференцирование экспоненты в (2.10) дает множитель $i H-L^{*} L / 2-L^{*} W v(t)$, мы получаем уравнение

$$
\left(\psi(w), d u_{t} \psi(v)\right)_{\Gamma^{S}}=\left(\psi(w),\left(u_{t+d t}-u_{t}\right) \psi(v)\right)_{\Gamma^{S}}=\left(\psi(w), u_{t} M(d t) \psi(v)\right)_{\Gamma^{S}}
$$

где

$$
\begin{aligned}
(\psi(w), M(d t) \psi(v))_{\Gamma^{S}}= & \left(\left(i H-L^{*} L / 2\right)+L \bar{w}(t)-L^{*} W v(t)\right. \\
& +(W-I) \bar{w}(t) v(t))(\psi(w), \psi(v))_{\Gamma^{S}} d t
\end{aligned}
$$

Учитьвая, что $(\psi(w), \Lambda(d t) \psi(v))_{\Gamma^{S}}=\overline{w(t)} v(t)(\psi(w), \psi(v))_{\Gamma^{S}} d t$ и

$$
\begin{aligned}
(\psi(w), A(d t) \psi(v))_{\Gamma^{S}} & =v(t)(\psi(w), \psi(v))_{\Gamma^{S}} d t \\
\left(\psi(w), A^{\dagger}(d t) \psi(v)\right)_{\Gamma^{S}} & =\overline{w(t)}(\psi(w), \psi(v))_{\Gamma^{S}} d t
\end{aligned}
$$

стохастический дифференциал $M(d t)$ может быть записан в стандартной форме Хадсона-Партасарати [6]-[8]:

$$
M(d t)=\left(i H-\frac{L^{*} L}{2}\right) d t-L^{*} W A(d t)+L A^{\dagger}(d t)+(W-I) \Lambda(d t)
$$

с коэффищиентами $H, K, R$ из теоремы 2.2. Таким образом, имеет место следующее утверждение.

ТЕорема 2.3. С точностью до унитарной эквивалентности, порождаемой операторами сдвига $e^{t \nabla}$, семейство унитарных разрешающих операторов (2.2) сходится в сильном смысле к решению квантового стохастического дифференциального уравнения $d u_{t}=u_{t} M(d t), t>0,\left.u\right|_{t=0}=I$, со стохастическим дифференциалом (2.11).

Аналогичные вычисления при $t<0$ для оператора эволюции (2.2) дают следующее выражение для стохастического дифференциала: $M(d t)=-\left(\left(W^{*}-I\right) \Lambda(d t)-\right.$ $\left.W^{*} L A^{\dagger}(d t)+L^{*} A(d t)-\left(i H+L^{*} L / 2\right) d t\right)$, т.е. $i M(-d t)=(i M(d t))^{*}$, что означает эквивалентность перехода к сопряженной эволюции изменению направления отсчета времени в уравнении Хадсона-Партасарати, рассматриваемому как уравнение Шрёдингера, 
а краевое условие (2.7) устанавливает связь между “прошлым" и “будущим" значением фазы в точке разрьва: $A\left(d t_{-}\right) \Psi_{t}=\left(W A\left(d t_{+}\right)+L d t\right) \Psi_{t}$; при этом фотоны, не достигшие зеркала, находятся в абсолютном будущем относительно фотонов, приобретающих сдвиг фазы в результате взаимодействия с зеркалом. Следовательно, наличие или отсутствие процесса измерения интенсивности излучения с помощью фотодетектора неможет влиять на эволюцию состояний квантового осциллятора.

Более интересным вьводом, которьй можно сделать, анализируя точное решение (2.4), касается квантового уравнения марковской эволюции (которое также назьвают квантовым уравнением Лиувиля, уравнением Горини-Сударшана-Коссаковского-Линдблада, квантовьм кинетическим уравнением, master Markov equation [9], [10]). Kaк мы увидим, чистые состояния электромагнитного поля, отвечающие различным экспоненциальным векторам, порождают различные операторы эволюции наблюдаемых квантовой системы из $\mathscr{B}(\mathscr{H})$. В результате оказьвается, что хотя не существует общего марковского уравнения в $\mathscr{B}(\mathscr{H})$, порождаемого суперпозицией чистых состояний поля, но эволюция наблюдаемых из $\mathscr{B}(\mathscr{H})$ равна суперпозиции эволюций относительно соответствующих набору чистых состояний.

Действительно, нетрудно видеть, что при $t>0$

$$
P_{t}(B) \stackrel{\text { def }}{=}\left(U_{t} \psi(v), B U_{t} \psi(v)\right)_{\Gamma^{S}}=B_{t}+\sum_{k=1}^{\infty} \frac{1}{k !} \int_{\mathbb{R}^{k}} d^{k} x\left(\prod_{k} \hat{v}_{t}\left(x_{k}\right)\right)^{*} B_{t}\left(\prod_{k} \hat{v}_{t}\left(x_{k}\right)\right),
$$

где $\hat{v}_{t}\left(x_{k}\right)=\left(W v\left(x_{k}+t\right)+L\right) I_{(-t, 0)}\left(x_{k}\right)+v(x+t) I_{\mathbb{R} \backslash(-t, 0)}\left(x_{k}\right)$ и для эрмитова оператора $B \in \mathscr{B}(\mathscr{H})$ имеем $B_{t}=\left(\exp \left\{\left(i H-L^{*} L / 2\right) t-L^{*} W \int_{0}^{t} v(s) d s\right\}\right)^{*} B\left(\exp \left\{\left(i H-L^{*} L / 2\right) t-\right.\right.$ $\left.\left.L^{*} W \int_{0}^{t} v(s) d s\right\}\right)$. Поэтому

$$
\begin{aligned}
\frac{d}{d t} \int_{\mathbb{R}} d x \hat{v}_{t}^{*}(x) B \hat{v}_{t}(x)= & \frac{d}{d t}\left(W^{*} B W \int_{0}^{t}|v(x)|^{2} d x\right. \\
& \left.+2 \operatorname{Re} L^{*} B W \int_{0}^{t} v(x) d x+B \int_{\mathbb{R} \backslash(0, t)}|v(x)|^{2} d x+L^{*} B L t\right) \\
= & (W v(t)+L)^{*} B(W v(t)+L)-|v(t)|^{2} B, \\
\frac{d}{d t} B_{t}= & -G_{t}^{*} B_{t}-B_{t} G_{t}, \quad G_{t}=i H-\frac{L^{*} L}{2}-L^{*} W v(t)-\frac{|v(t)|^{2}}{2} .
\end{aligned}
$$

Таким образом, выражение для генератора $\mathscr{L}_{t}(\cdot)$ вполне положительной эволюции имеет вид, зависящий от аргумента экспоненциального вектора: $\mathscr{L}_{t}(B)=\Phi_{t}(B)-G_{t}^{*} B-$ $B G_{t}, \Phi_{t}(B)=(W v(t)+L)^{*} B(W v(t)+L), G_{t}=i H-L^{*} L / 2-L^{*} W v(t)-|v(t)|^{2} / 2$.

Более общий результат, не предполагающий коммутативность операторов $K, L, R$, можно получить, используя следующую формулу интегрирования по частям (см. [5]) для фоковских векторов $\Gamma^{S}\left(W_{2}^{1}(\mathbb{R} \backslash\{0\})\right)$, удовлетворяющих краевому условию (2.7):

$$
\begin{aligned}
2 \operatorname{Re}(\psi, B \nabla \varphi)_{\Gamma^{S}} & =\left(A_{-} \psi, B A_{-} \varphi\right)_{\Gamma^{S}}-\left(A_{+} \psi, B A_{+} \varphi\right)_{\Gamma^{S}} \\
& =\left(\left(L+W A_{+}\right) \psi, B\left(L+W A_{+}\right) \varphi\right)_{\Gamma^{S}}-\left(A_{+} \psi, B A_{+} \varphi\right)_{\Gamma^{S}} .
\end{aligned}
$$


ТЕорема 2.4. Предположим, что начальное состояние әлектромагнитного поля имеет вид смешанного состояния $\rho=\sum_{n} \rho_{n}, \rho_{n}=r_{n}\left|\psi\left(v_{n}\right)\right\rangle\left\langle\psi\left(v_{n}\right)\right|, \quad \operatorname{Tr} \rho=1$, $u$ пусть $U_{t}$ - унитарная группа, удовлетворяющая уравнению (2.8) с произвольными ограниченными операторными коәффичиентами $K, R, L$ и краевому условию (2.7). Тогда среднее по состоянию поля (частичный след относительно ортогонального базиса в фоковском пространстве)

$$
\begin{aligned}
P_{t}(B) & =\operatorname{Tr}_{\Gamma}\left\{(\rho \otimes I) U_{t}^{*}(I \otimes B) U_{t}\right\} \\
& =\sum_{n} r_{n} \operatorname{Tr}_{\Gamma} S\left\{\left(\rho_{n} \otimes I\right) U_{t}^{*}(I \otimes B) U_{t}\right\}=\sum_{n} r_{n} P_{t}^{(n)}(B)
\end{aligned}
$$

является суперпозииией решений квантовых уравнений Лиувилля $\frac{d}{d t} P_{t}^{(n)}(B)=$ $P_{t}^{(n)}\left(\mathscr{L}_{t}^{(n)}(B)\right)$, генераторы которых в форме Линдблада имеют вид

$$
\mathscr{L}_{t}^{(n)}(B)=\Phi_{t}^{(n)}(B)-\left(G_{t}^{(n)}\right)^{*} B-B G_{t}^{(n)}, \quad G_{t}^{(n)}=i H-\frac{1}{2} \Phi_{t}^{(n)}(I)
$$

əде $\Phi_{t}^{(n)}(B)=\left(L+W v_{n}(t)\right)^{*} B\left(L+W v_{n}(t)\right)$.

ДокАЗАТЕЛьСтво. В силу принципа суперпозиции, доказательство достаточно провести для одного слагаемого $\rho_{n}=\left|\psi\left(v_{n}\right)\right\rangle\left\langle\psi\left(v_{n}\right)\right|$. Пусть $B \in \mathscr{B}(\mathscr{H})$ и $v_{n} \in W_{2}^{1}(\mathbb{R})$. Тогда из (2.13) и равенства $A_{+} U_{t} \Psi\left(v_{n}\right)=v(t) U_{t} \Psi\left(v_{n}\right)$ следует

$$
\begin{aligned}
\frac{d}{d t} P_{t}^{(n)}(B)= & \frac{d}{d t}\left(U_{t} \psi\left(v_{n}\right), B U_{t} \psi\left(v_{n}\right)\right)_{\Gamma^{S}}=2 \operatorname{Re}\left(\nabla U_{t} \psi\left(v_{n}\right), B U_{t} \psi\left(v_{n}\right)\right)_{\Gamma^{S}} \\
& +\operatorname{Re}\left(\left(i H-L^{*} L / 2+L^{*} W A_{+}\right) U_{t} \psi\left(v_{n}\right), B U_{t} \psi\left(v_{n}\right)\right)_{\Gamma^{S}} \\
= & \left(U_{t} \psi\left(v_{n}\right),\left(L+W v_{n}(t)\right)^{*} B\left(L+W v_{n}(t)\right) U_{t} \psi\left(v_{n}\right)\right)_{\Gamma^{S}} \\
& -\left(U_{t} \psi\left(v_{n}\right),\left|v_{n}(t)\right|^{*} B U_{t} \psi\left(v_{n}\right)\right)_{\Gamma^{S}} v_{n}(t) \\
& +\operatorname{Re}\left(U_{t} \psi\left(v_{n}\right), B\left(i H-L^{*} L / 2+L^{*} W v_{n}(t)\right) U_{t} \psi\left(v_{n}\right)\right)_{\Gamma^{S}} \\
= & \left(U_{t} \psi\left(v_{n}\right), \mathscr{L}_{t}^{(n)}(B) U_{t} \psi\left(v_{n}\right)\right)_{\Gamma^{S}} .
\end{aligned}
$$

Теорема доказана.

3. Сильный резольвентный предел: гамильтониан с некоммутирующими коэффициентами. Построим точное решение $\psi_{t}$ задачи Коши для уравнения Шрёдингера

$$
\frac{1}{i} \frac{d}{d t} \psi_{t}=\mathbf{H}_{t}^{\alpha} \psi_{t}, \quad \psi_{t}=U_{t, 0}^{\alpha} \psi
$$

с гамильтонианом

$\mathbf{H}_{t}^{\alpha}=H_{0}+H_{\mathrm{int}}, \quad H_{0}=\Omega b^{\dagger} b-i \nabla, \quad H_{\mathrm{int}}=\mu\left(b^{\dagger}+b\right)\left(\int_{\mathbb{R}} g_{\alpha}(x) a^{\dagger}(x) a(x) d x+f_{t}\right)$.

Нетрудно видеть, что коэффициенты $H_{0}$ и $H_{\text {int }}$ не коммутируют, а гамильтониан $\mathbf{H}_{t}^{\alpha}$ является регуляризацией сингулярного гамильтониана (1.2) при $\hbar=1, c=1, \mu=$ 
$\hbar \omega g / \sqrt{2}, f_{t}=(g / \mu \sqrt{2}) f(t)$. В представление взаимодействия, порождаемом оператором $H_{0}$, уравнение Шрёдингера для $\varphi_{t}=e^{-i H_{0} t} \psi_{t}$ имеет вид

$\frac{\partial}{\partial t} \varphi_{t}=i e^{-i H_{0} t} H_{\mathrm{int}} e^{i H_{0} t} \varphi_{t}=i \mu\left(b_{t}^{\dagger}+b_{t}\right) F_{t}^{\alpha} \varphi_{t}, \quad F_{t}^{\alpha}=\int_{\mathbb{R}} g_{\alpha}(x-t) a^{\dagger}(x) a(x) d x+f_{t}$,

где $b_{t}=b e^{i \Omega t}, b_{t}^{\dagger}=b^{\dagger} e^{-i \Omega t}$, а операторы $F_{s}^{\alpha}$ и $F_{\tau}^{\alpha}$ коммутируют при различных $\tau$ и $s$. Поэтому разрешающий оператор в представлении взаимодействия может быть получен в виде произведения трех экспонент:

$$
\begin{gathered}
\varphi_{t}=V_{(0, t)}^{\alpha} \varphi, \quad V_{T}^{\alpha}=e^{-\mu^{2} C_{T}^{\alpha}} e^{i \mu b^{\dagger} \bar{\beta}_{T}^{\alpha}} e^{i \mu b \beta_{T}^{\alpha}}, \\
\beta_{T}^{\alpha}=\int_{T} d s e^{i \Omega s} F_{s}^{\alpha}, \quad \bar{\beta}_{T}^{\alpha}=\int_{T} d s e^{-i \Omega s} F_{s}^{\alpha}, \\
C_{T}^{\alpha}=\frac{1}{2} \int_{T} d s F_{s}^{\alpha} \int_{T} d \tau e^{i|\Omega(s-\tau)|} F_{\tau}^{\alpha},
\end{gathered}
$$

где $T \subseteq \mathbb{R}_{+}$- любое борелевское множество. Операторы $V^{\alpha}$ сохраняют $n$-частичные подпространства фоковского пространства, что значительно упрошает обоснование слабых предельных переходов.

Оператор $e^{-\mu^{2} C_{T}^{\alpha}}$ коммутирует с остальным сомножителями в $(3.2)$ и, более того, нетрудно видеть, что

$$
\bar{\beta}_{T}^{\alpha} \beta_{T}^{\alpha}=\bar{C}_{T}^{\alpha}+C_{T}^{\alpha}, \quad \beta_{T}^{\alpha} \bar{\beta}_{S}^{\alpha}+C_{T}^{\alpha}+C_{S}^{\alpha}=C_{S \cup T}^{\alpha},
$$

где $T$ и $S$ - непересекаюшиеся борелевские множества такие, что $t>s$ для любых $t \in T$, $s \in S$. Отсюда следует, что

$$
C_{T}^{\alpha}-\frac{\bar{\beta}_{T}^{\alpha} \beta_{T}^{\alpha}}{2}=\frac{C_{T}^{\alpha}-\bar{C}_{T}^{\alpha}}{2}=i \operatorname{Im} C_{T}^{\alpha}=\frac{i}{2} \int_{T} \int_{T} d s d \tau F_{s}^{\alpha} F_{\tau}^{\alpha} \sin |\Omega(\tau-s)| .
$$

Поэтому разрешающие операторы $V_{T}$ и $U_{t}$ могут быть записаны в виде

$$
\begin{aligned}
V_{T} & =e^{-\mu^{2} C_{T}^{\alpha}} e^{i \mu b^{\dagger} \bar{\beta}_{T}^{\alpha}} e^{i \mu b \beta_{T}^{\alpha}}=\exp \left\{i \mu\left(b^{\dagger} \bar{\beta}_{T}^{\alpha}+b \beta_{T}^{\alpha}\right)-i \mu^{2} \operatorname{Im} C_{T}^{\alpha}\right\} \\
U_{t} & =e^{i H_{0} t} e^{-\mu^{2} C_{(0, t)}^{\alpha}} e^{i \mu b^{\dagger} \bar{\beta}_{(0, t)}^{\alpha}} e^{i \mu b \beta_{(0, t)}^{\alpha}} \\
& =e^{i H_{0} t} \exp \left\{i \mu\left(b^{\dagger} \bar{\beta}_{(0, t)}^{\alpha}+b \beta_{(0, t)}^{\alpha}\right)-i \mu^{2} \operatorname{Im} C_{(0, t)}^{\alpha}\right\}
\end{aligned}
$$

Правые части обеих формул являются композициями унитарных операторов, поэтому $U_{t}$ и $V_{T}$ - семейства унитарных операторов. Покажем, что в случае $f_{t}=$ const семейство $U_{t}$ является группой.

Заметим, что в силу известных коммутационных соотношений [11] справедливы следующие тождества:

$$
\begin{gathered}
e^{-i s \Omega b^{\dagger} b} b e^{i s \Omega b^{\dagger} b}=b e^{i s \Omega}, \quad e^{\bar{\nu} b^{\dagger}+\nu b} e^{\mu b^{\dagger}+\bar{\mu} b}=e^{(\nu+\mu) b^{\dagger}+(\bar{\nu}+\bar{\mu}) b} e^{(\nu \bar{\mu}-\mu \bar{\nu}) / 2} \\
e^{-s \nabla} a^{\dagger}(x) a(x) e^{s \nabla}=a^{\dagger}(x+s) a(x+s) .
\end{gathered}
$$


Поэтому вьполнены равенства

$$
\begin{gathered}
e^{-i H_{0} s} b \beta_{T}^{\alpha} e^{i H_{0} s}=b \beta_{T+s}^{\alpha}, \quad e^{-i H_{0} s} C_{T}^{\alpha} e^{i H_{0} s}=C_{T+s}^{\alpha} \\
e^{i \bar{\beta}_{T}^{\alpha} b^{\dagger}+i \beta_{T}^{\alpha} b} e^{i \bar{\beta}_{S}^{\alpha} b^{\dagger}+i \beta_{S}^{\alpha} b}=e^{i \bar{\beta}_{T}^{\alpha} \cup S^{\dagger}+i \beta_{T \cup S}^{\alpha} b} e^{\left(\bar{\beta}_{S}^{\alpha} \beta_{T}^{\alpha}-\bar{\beta}_{T}^{\alpha} \beta_{S}^{\alpha}\right) / 2} \\
\frac{d}{d t} e^{i\left(\beta_{t} b+\bar{\beta}_{t} b^{\dagger}\right)}=\left\{i\left(\beta_{t}^{\prime} b+\bar{\beta}_{t}^{\prime} b^{\dagger}\right)+\frac{1}{2}\left(\bar{\beta}_{t} \beta_{t}^{\prime}-\beta_{t} \bar{\beta}_{t}^{\prime}\right)\right\} e^{i\left(\beta_{t} b+\bar{\beta}_{t} b^{\dagger}\right)}
\end{gathered}
$$

Полагая $T=(s, s+t), S=(0, s)$ и используя первые два равенства $(3.4)$, получаем групповое свойство семейства $U_{t}$ :

$$
\begin{aligned}
U_{s} U_{t}= & e^{i H_{0}(t+s)} e^{i \mu\left(b^{\dagger} \bar{\beta}_{(s, t+s)}^{\alpha}+b \beta_{(s, t+s)}^{\alpha}\right)-i \mu^{2} \operatorname{Im} C_{(s, t+s)}^{\alpha}} e^{i \mu\left(b^{\dagger} \bar{\beta}_{(0, s)}^{\alpha}+b \beta_{(0, s)}^{\alpha}\right)-i \mu^{2} \operatorname{Im} C_{(0, s)}^{\alpha}} \\
= & e^{i(t+s) H_{0}} e^{i \mu\left(b^{\dagger} \bar{\beta}_{(0, t+s)}^{\alpha}+b \beta_{(0, t+s)}^{\alpha}\right)} \\
& \times e^{-i \mu^{2} \operatorname{Im}\left(C_{(0, s)}^{\alpha}+C_{(s, t+s)}^{\alpha}\right)+\mu^{2}\left(\beta_{(0, s)}^{\alpha} \bar{\beta}_{(s, t+s)}^{\alpha}-\beta_{(s, t+s)}^{\alpha} \bar{\beta}_{(0, s)}^{\alpha}\right) / 2}=U_{t+s} .
\end{aligned}
$$

Обозначим через $\pi_{n}$ проектор на $n$-частичное подпространство симметричного пространства $\Phi$ ока $\Gamma^{S}$ и напомним правила действия оператора числа частиц:

$$
\begin{gathered}
\pi_{n} \int_{\mathbb{R}} g_{\alpha}(x-t) a^{\dagger}(x) a(x) d x \psi(x)=\sum_{k=1}^{n} g_{\alpha}\left(x_{k}-t\right) \psi_{n}\left(x_{1}, \ldots, x_{n}\right), \\
\pi_{n} \int_{\mathbb{R}} g_{\alpha}(y-t) a^{\dagger}(y) a(y) d y \int_{\mathbb{R}} g_{\alpha}(z-s) a^{\dagger}(z) a(z) d z \psi(x) \\
=\sum_{k, j=1}^{n} g_{\alpha}\left(x_{k}-t\right) g_{\alpha}\left(x_{j}-s\right) \psi_{n}(x), \\
\pi_{n} e^{\int_{\mathbb{R}} a^{\dagger}(y) a(y) d y \int_{\mathbb{R}} R(y, z) a^{\dagger}(z) a(z) d z} \psi(x)=\psi_{n}\left(x_{1}, \ldots, x_{n}\right) e^{\sum_{k, j=1}^{n} R\left(x_{k}, x_{j}\right)} .
\end{gathered}
$$

С помощью этих соотношений нетрудно убедиться, что если $\alpha \rightarrow 0$ и $g_{\alpha} \rightarrow \delta(x)$, то существуют пределы в $n$-частичных подпространствах, которые при $t>0$ принимают следуюшие значения:

$$
\begin{aligned}
& \beta_{T}^{\alpha} \stackrel{w}{\longrightarrow} \beta_{T} \stackrel{\text { def }}{=} \int d x I_{(0, t)}(x) e^{i \Omega x}\left(a^{\dagger}(x) a(x)+f_{x}\right),
\end{aligned}
$$

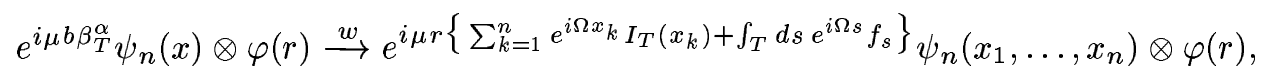

$$
\begin{aligned}
& C_{T}^{\alpha} \stackrel{w}{\longrightarrow} C_{T} \stackrel{\text { def }}{=} \frac{1}{2} \int_{\mathbb{R}} d x \int_{\mathbb{R}} d y I_{T}(x) I_{T}(y)\left(a^{\dagger}(x) a(x)+f_{x}\right)\left(a^{\dagger}(y) a(y)+f_{y}\right) \sin |\Omega(x-y)|, \\
& e^{-i \mu^{2} \operatorname{Im} C_{T}^{\alpha}} \psi_{n}(x) \stackrel{w}{\longrightarrow} e^{-i \mu^{2} \operatorname{Im} C_{T}} \psi_{n}(x)=\exp \left\{-\frac{i \mu^{2}}{2}\left(\int_{T} d s f_{s} \int_{T} d \tau f_{\tau} \sin |\Omega(\tau-s)|\right.\right. \\
& +2 \sum_{k=1}^{n} I_{T}\left(x_{k}\right) \int_{T} d s f_{s} \sin \left|\Omega\left(s-x_{k}\right)\right| \\
& \left.\left.+\sum_{k, j=1}^{n} I_{T}\left(x_{k}\right) I_{T}\left(x_{j}\right) \sin \left|\Omega\left(x_{j}-x_{k}\right)\right|\right)\right\} \psi_{n}(x)
\end{aligned}
$$


где $\varphi(r)$ - экспоненциальньй вектор в $\ell_{2}(b \varphi(r)=r \varphi(r) \forall r \in \mathbb{C})$. Переходя к слабому пределу при $\alpha \rightarrow 0$ в $n$-частичных подпространствах, мы видим, что существуют слабые пределы семейств унитарных операторов, которые сами являются унитарными операторами:

$$
\begin{aligned}
V_{T} & =w-\lim _{\alpha \rightarrow 0} e^{-\mu^{2} C_{T}^{\alpha}} e^{i \mu b^{\dagger} \bar{\beta}_{T}^{\alpha}} e^{i \mu b \beta_{T}^{\alpha}}=\exp \left\{i \mu\left(b^{\dagger} \bar{\beta}_{T}+b \beta_{T}\right)-i \mu^{2} \operatorname{Im} C_{T}\right\} \\
U_{t} & =w-\lim _{\alpha \rightarrow 0} e^{i H_{0} t} e^{-\mu^{2} C_{(0, t)}^{\alpha}} e^{i \mu b^{\dagger} \bar{\beta}_{(0, t)}^{\alpha}} e^{i \mu b \beta_{(0, t)}^{\alpha}} \\
& =e^{i H_{0} t} \exp \left\{i \mu\left(b^{\dagger} \bar{\beta}_{(0, t)}+b \beta_{(0, t)}\right)-i \mu^{2} \operatorname{Im} C_{(0, t)}\right\}
\end{aligned}
$$

и унитарньй коцикл

$$
u_{(t, s)} \stackrel{\text { def }}{=} e^{i \Omega b^{\dagger} b t} V_{(s, t)} e^{-i \Omega b^{\dagger} b s}=e^{i \Omega b^{\dagger} b t} e^{i \mu\left(b^{\dagger} \bar{\beta}_{(s, t)}+b \beta_{(s, t)}\right)-i \mu^{2} \operatorname{Im} C_{(s, t)}} e^{-i \Omega b^{\dagger} b s} .
$$

Сильная сходимость к пределу следует из слабой сходимости операторов в $n$-частичных подпространствах и очевидной унитарности слабых пределов. Сильная непрерывность унитарной групы по времени следует из слабой непрерьвности. Свойство коцикличности семейства $u_{(t, \tau)} u_{(\tau, s)}=u_{(t, \tau)}$ следует из правила коммутации

$$
e^{i\left(\nu b^{\dagger}+\bar{\nu} b\right)} e^{i\left(\mu b^{\dagger}+\bar{\mu} b\right)}=e^{i(\nu+\mu) b^{\dagger}+i(\overline{\nu+\mu}) b} e^{-i \operatorname{Im} \mu \bar{\nu}}
$$

и второго соотношения (3.3), которое остается справедливым для предела операторов (3.4).

Выведем эволюционное уравнение и краевое условие для семейства операторов $U_{t}$. Согласно (3.7)

$$
\left(U_{t} \psi(v)\right)_{n}(x)=\exp \left\{i \Omega b^{\dagger} b t\right\} \frac{1}{\sqrt{n} !} e^{\left\{i \mu \widehat{S}_{n}(x, t)-\frac{i \mu^{2}}{2} \Phi_{n}(x, t)\right\}} \prod_{1}^{n} v\left(x_{k}+t\right)
$$

где $x \in \mathbb{R}^{n}, \widehat{S}_{n}(x, t)=b\left(\beta_{n}(x, t)+\phi(t)\right)+b^{\dagger}\left(\bar{\beta}_{n}(x, t)+\bar{\phi}(t)\right)$,

$$
\begin{aligned}
\beta_{n}(x, t) & =\sum_{k=1}^{n} e^{i \Omega\left(x_{k}+t\right)} I_{(-t, 0)}\left(x_{k}\right), \quad \phi(t)=\int_{0}^{t} f_{s} e^{i \Omega s} d s \\
\Phi_{n}(x, t)= & \int_{0}^{t} \int_{0}^{t} d s d \tau f_{s} f_{\tau} \sin |\Omega(\tau-s)| \\
& +2 \sum_{k=1}^{n} I_{(-t, 0)}\left(x_{k}+t\right) \int_{0}^{t} d s f_{s} \sin \left|\Omega\left(x_{k}+t-s\right)\right| \\
& +\sum_{k, j=1}^{n} I_{(-t, 0)}\left(x_{k}+t\right) I_{(-t, 0)}\left(x_{j}+t\right) \sin \left|\Omega\left(x_{j}-x_{k}\right)\right|
\end{aligned}
$$


Обозначим $\nabla_{n}=\sum_{k=1}^{n} \partial_{x_{k}}$. Нетрудно видеть, что вьполнены следующие равенства:

$$
\begin{gathered}
\left(\partial_{t}-\nabla_{n}\right)\left(\beta_{n}(x, t)+\phi(t)\right)=\phi^{\prime}(t)=f_{t} e^{i \Omega t}, \quad\left(\partial_{t}-\nabla_{n}\right) \prod_{1}^{n} v\left(x_{k}+t\right)=0 \\
\left(\partial_{t}-\nabla_{n}\right) I_{(-t, 0)}(x)=0, \quad\left(\partial_{t}-\nabla_{n}\right) \Phi_{n}(x, t)=2 \phi_{n}(x, t), \quad x \neq 0 \\
\phi_{n}(x, t)=\left(\int_{0}^{t} f_{s} \sin \Omega(t-s) d s-2 \sum_{k=1}^{n} I_{(-t, 0)}\left(x_{k}\right) \sin \Omega x_{k}\right) f_{t} \\
=\frac{1}{2 i}\left(\left(\phi^{\prime}(t) \bar{\beta}_{n}(x, t)+\bar{\phi}(t)\right)-\bar{\phi}^{\prime}(t)\left(\beta_{n}(x, t)+\phi(t)\right)\right) .
\end{gathered}
$$

Применяя последнюю формулу (3.4) для дифференцирования операторной экспоненты $\exp \left\{i \mu \widehat{S}_{n}(x, t)\right\}$, получим

$$
\begin{aligned}
\left(\partial_{t}-\nabla_{n}\right) e^{i \mu \widehat{S}_{n}(x, t)}= & \left\{i \mu\left(b e^{i \Omega t}+b^{\dagger} e^{-i \Omega t}\right) f_{t}\right. \\
& \left.+\frac{\mu^{2}}{2}\left(\left(\phi^{\prime}(t) \bar{\beta}_{n}(x, t)+\bar{\phi}(t)\right)-\bar{\phi}^{\prime}(t)\left(\beta_{n}(x, t)+\phi(t)\right)\right)\right\} e^{i \mu \widehat{S}_{n}(x, t)} \\
= & \left\{i \mu\left(b e^{i \Omega t}+b^{\dagger} e^{-i \Omega t}\right) f_{t}+i \mu^{2} \phi_{n}(x, t)\right\} e^{i \mu \widehat{S}_{n}(x, t)} .
\end{aligned}
$$

Отсюда и из (3.10), (3.11) имеем

$$
\begin{gathered}
\left(\partial_{t}-\nabla_{n}\right) e^{i \mu \widehat{S}_{n}(x, t)-\frac{i \mu^{2}}{2} \Phi_{n}(x, t)}=\left\{i \mu\left(b e^{i \Omega t}+b^{\dagger} e^{-i \Omega t}\right) f_{t}\right\} e^{i \mu \widehat{S}_{n}(x, t)-\frac{i \mu^{2}}{2} \Phi_{n}(x, t)}, \\
\left(\partial_{t}-\nabla_{n}\right) e^{i \Omega b^{\dagger} b t} \prod_{1}^{n} v\left(x_{k}+t\right)=i \Omega b^{\dagger} b e^{i \Omega b^{\dagger} b t} \prod_{1}^{n} v\left(x_{k}+t\right) .
\end{gathered}
$$

Учитьвая, что $e^{i \Omega b^{\dagger} b t}\left(b e^{i \Omega t}+b^{\dagger} e^{-i \Omega t}\right) e^{-i \Omega b^{\dagger} b t}=b+b^{\dagger}$, мы получаем уравнение

$$
\left(\frac{\partial}{\partial t}-\nabla\right) U_{t} \psi(v)=\left(i \Omega b^{\dagger} b+i \mu\left(b+b^{\dagger}\right) f_{t}\right) U_{t} \psi(v)
$$

справедливое вне координатных гиперплоскостей $x=0$ для любого экспоненциального вектора $\psi(v), v \in W_{2}^{1}(\mathbb{R})$.

Получим условие для разрывов решения на координатных гиперплоскостях. Из определения функций $\beta_{n}(x, t)$ и $\Phi_{n}(x, t)$ следуют равенства

$$
\begin{gathered}
\left.\beta_{n+1}(x, t)\right|_{x_{n+1}=+0}=\beta_{n}(x, t),\left.\quad \beta_{n+1}(x, t)\right|_{x_{n+1}=-0}=\beta_{n}(x, t)+e^{i \Omega t}, \\
\left.\Phi_{n+1}(x, t)\right|_{x_{n+1}=+0}=\Phi_{n}(x, t), \\
\left.\Phi_{n+1}(x, t)\right|_{x_{n+1}=-0}=\Phi_{n}(x, t)+2 \int_{0}^{t} f_{s} \sin \Omega(t-s) d s-2 \sum_{k=1}^{n} I_{(-t, 0)}\left(x_{k}\right) \sin \Omega x_{k} \\
=\Phi_{n}(x, t)+i e^{i \Omega t}\left(\bar{\beta}_{n}(x, t)+\bar{\phi}(t)\right)-i e^{-i \Omega t}\left(\beta_{n}(x, t)+\phi(t)\right) .
\end{gathered}
$$


Теперь нетрудно видеть, что $\left(A_{+} U_{t} \psi(v)\right)_{n}(x)=v(t)\left(U_{t} \psi(v)\right)_{n}(x)$. С другой стороны, выпутывая согласно (3.9) и (3.13) член $e^{i \mu\left(b e^{i \Omega t}+b^{\dagger} e^{-i \Omega t}\right)}$ из операторной экспоненты $\exp \left\{i \mu\left(b\left(\beta_{n}(x, t)+\phi(t)+e^{i \Omega t}\right)+b^{\dagger}\left(\bar{\beta}_{n}(x, t)+\bar{\phi}(t)+e^{-i \Omega t}\right)\right)\right\}$ получаем

$$
\begin{gathered}
\left(A_{-} U_{t} \psi(v)\right)_{n}(x)=\frac{v(t)}{\sqrt{n !}} e^{i \Omega b^{\dagger} b t} e^{i \mu\left(b\left(\beta_{n}(x, t)+\phi(t)+e^{i \Omega t}\right)+b^{\dagger}\left(\bar{\beta}_{n}(x, t)+\bar{\phi}(t)+e^{-i \Omega t}\right)\right)} \\
\times e^{-\frac{i \mu^{2}}{2}\left(\Phi_{n}(x, t)+2 \int_{0}^{t} f_{s} \sin \Omega(t-s) d s-2 \sum_{k=1}^{n} I_{(-t, 0)}\left(x_{k}\right) \sin \Omega x_{k}\right)} \prod_{1}^{n} v\left(x_{k}+t\right) \\
=v(t) e^{i \Omega b^{\dagger} b t} e^{i \mu\left(b e^{i \Omega t}+b^{\dagger} e^{-i \Omega t}\right)} e^{-i \Omega b^{\dagger} b t}\left(U_{t} \psi(v)\right)_{n}(x)=e^{i \mu\left(b+b^{\dagger}\right)}\left(U_{t} \psi(v)\right)_{n}(x) .
\end{gathered}
$$

Следовательно, граничное условие имеет вид $A_{-} \psi=W A_{+} \psi, W=e^{i \mu\left(b+b^{\dagger}\right)}$; оно описывает скачок фазы пучка когерентных фотонов при взаимодействии с зеркалом, установленном на квантовом осцилляторе. Таким образом, доказана первая часть следующего утверждения.

ТЕОРема 3.1. Сильный резольвентный предел семейства уравнений Шрёдингера с гамильтонианами (3.1) является краевой задачей

$$
\begin{gathered}
\frac{d}{d t} \psi_{t}=\left(\nabla+i \Omega b^{\dagger} b+i \mu\left(b^{\dagger}+b\right) f_{t}\right) \psi_{t}, \quad A_{-} \psi_{t}=W A_{+} \psi_{t}, \\
W=e^{i \mu\left(b^{\dagger}+b\right)}, \quad \psi_{t}=U_{t} \psi_{0} .
\end{gathered}
$$

Унитарный кочикл $u_{t}=e^{-\nabla t} U_{t}$ в слабом смысле удовлетворяет квантовому стохастическому дифференииальному уравнению

$$
d u_{t}=M\left(d t_{+}\right) u_{t}, \quad M(T)=i \int_{T}\left(\Omega b^{\dagger} b+\mu\left(b^{\dagger}+b\right) f_{t}\right) d t+(W-I) \Lambda(T),
$$

əде $\Lambda(T)=\int_{T} a^{\dagger}(t) a(t) d t$.

Наиболее простой вывод уравнения (3.15) состоит в использовании уравнения (3.14) и формулы интегрирования по частям (2.13). Напомним, что обозначение $M\left(d t_{+}\right)$указьвает, что стохастический дифференциал понимается в смыслеИто, а не Стратоновича (см. [12]), т.е. для любого экспоненциального вектора $\psi(v), v \in C_{0}^{\infty}(\mathbb{R})$, вьполнено равенство, являющееся слабой формой уравнения (3.15):

$$
\frac{d}{d t}\left(\psi(v), \Psi_{t}\right)=i\left(\Omega b^{\dagger} b+\mu\left(b^{\dagger}+b\right) f_{t}\right)\left(\psi(v), \Psi_{t}\right)+(W-I)\left(A_{+}(t) \psi(v), A_{+}(t) \Psi_{t}\right),
$$

где $\Psi_{t}=u_{t} \psi_{0}, A_{ \pm}(t)=e^{-t \nabla} A_{ \pm} e^{t \nabla}, A_{ \pm}(t) \psi(h)=h(t \pm 0) \psi(h)$ для любой кусочно непрерьвной функции $h \in L_{2}(\mathbb{R})$. С другой стороны, применяя формулу $(2.13)$, получим

$$
\begin{aligned}
\frac{d}{d t}\left(\psi(v), e^{-t \nabla} U_{t} \psi_{0}\right)= & \frac{d}{d t}\left(e^{t \nabla} \psi(v), U_{t} \psi_{0}\right)=\left(\nabla e^{t \nabla} \psi(v), U_{t} \psi_{0}\right)+\left(e^{t \nabla} \psi(v), \nabla U_{t} \psi_{0}\right) \\
& +i\left(\Omega b^{\dagger} b+\mu\left(b^{\dagger}+b\right) f_{t}\right)\left(e^{t \nabla} \psi(v), U_{t} \psi_{0}\right) \\
= & \left(A_{-} \psi_{t}(v), A_{-} \psi_{t}\right)-\left(A_{+} \psi_{t}(v), A_{+} \psi_{t}\right) \\
& +i\left(\Omega b^{\dagger} b+\mu\left(b^{\dagger}+b\right) f_{t}\right)\left(\psi_{t}(v), \psi_{t}\right)
\end{aligned}
$$


где $\psi_{t}=U_{t} \psi_{0}, \psi_{t}(v)=e^{t \nabla} \psi(v), A_{ \pm} \psi_{t}(v)=v(t) \psi_{t}(v)$, и в силу граничного условия (3.14) $A_{-} \psi_{t}=W A_{+} \psi_{t}$. Учитьвая очевидные тождества $\left(\psi_{t}(v), \psi_{t}\right)=\left(\psi(v), \Psi_{t}\right)$, $\left(A_{+} \psi_{t}(v), A_{+} \psi_{t}\right)=\left(A_{+}(t) \psi(v), A_{+}(t) \Psi_{t}\right)$, видим, что

$$
\left(A_{-} \psi_{t}(v), A_{-} \psi_{t}\right)-\left(A_{+} \psi_{t}(v), A_{+} \psi_{t}\right)=(W-I)\left(A_{+}(t) \psi(v), A_{+}(t) \Psi_{t}\right)
$$

Таким образом, уравнение (3.16) совпадает со слабой формой краевой задачи (3.14). Вторая часть теоремы доказана.

Отметим, что из структуры квантового стохастического дифференциального уравнения не очевидны краевые условия (2.7), (3.14). С этим обстоятельством были связаны технические трудности при построении области определения стохастического дифференциала с неограниченными операторными коэффициентами, инвариантной относительно разрешающего оператора, соответствующего КСДУ [13]-[15].

В заключение сформулируем важное следствие теоремы 3.1 о конструкции уравнения для частичного следа по смешанному состоянию излучения лазера.

Теорема 3.2. Предположим, что начальное состояние әлектромагнитного поля имеет вид смешанного состояния $\rho=\sum_{n} \rho_{n}, \rho_{n}=r_{n}\left|\psi\left(v_{n}\right)\right\rangle\left\langle\psi\left(v_{n}\right)\right|, \quad \operatorname{Tr} \rho=$ 1, и пусть $U_{t}$ - семейство унитарных операторов, удовлетворяющих уравнению (3.14). Тогда среднее по состоянию поля

$$
\begin{aligned}
P_{t}(B) & =\operatorname{Tr}_{\Gamma S}\left\{(\rho \otimes I) U_{t}^{*}(I \otimes B) U_{t}\right\} \\
& =\sum_{n} r_{n} \operatorname{Tr}_{\Gamma}\left\{\left(\rho_{n} \otimes I\right) U_{t}^{*}(I \otimes B) U_{t}\right\}=\sum_{n} r_{n} P_{t}^{(n)}(B)
\end{aligned}
$$

является суперпозицией решений квантовых уравнений Лиувилля

$$
\frac{d}{d t} P_{t}^{(n)}(B)=P_{t}^{(n)}\left(\mathscr{L}_{t}^{(n)}(B)\right)
$$

генераторы которых в форме Линдблада имеют вид

$$
\mathscr{L}_{t}^{(n)}(B)=\Phi_{t}^{(n)}(B)-\left(G_{t}^{(n)}\right)^{*} B-B G_{t}^{(n)}, \quad G_{t}^{(n)}=i \Omega b^{\dagger} b+i \mu\left(b^{\dagger}+b\right) f_{t}-\frac{\left|v_{n}(t)\right|^{2}}{2}
$$

əде $\Phi_{t}^{(n)}(B)=\left|v_{n}(t)\right|^{2} W^{*} B W$

Доказательство этого утверждения основано на использовании формулы интегрирования по частям (2.13) и полностью аналогично доказательству теоремы 2.4. Оба эти утверждения показывают, что для точно решаемых задач, рассмотренных в настоящей статье, не существует уравнения типа уравнения Линдблада для эволюции частичного следа; напротив, каждая компонента смеси эволюционирует по собственному закону. 


\section{СПИСОК ЦИТИРОВАННОЙ ЛИТЕРАТУРЫ}

[1] Albeverio S., Karwowski W., Koshmanenko V. Square powers of singularly perturbed operators // Math. Nachr. 1995. V. 173. P. 5-24.

[2] Чеботарев А. М. Квантовое стохастическое дифференциальное уравнение унитарно эквивалентно краевой задаче для уравнения Шрёдингера // Матем. заметки. 1997. Т. 61. № 4. C. $612-622$.

[3] Chebotarev A. M. Lectures on Quantum Probability. V. 14. México: Sociedad Matemática Mexicana, Aportaciones Matemáticas, 2000.

[4] Като Т. Теория возмущений линейных операторов. М.: Наука, 1976.

[5] Чеботарев А. М. Что такое квантовое стохастическое уравнение с точки зрения функционального анализа // Матем. заметки. 2002. Т. 71. № 3. С. 448-469.

[6] Hudson R. L., Parthasarathy K. R. Quantum Ito's formula and stochastic evolutions // Comm. Math. Phys. 1984. V. 93. №3. P. 301-323.

[7] Parthasarathy K. R. An Introduction to Quantum Stochastic Calculus. Basel: Birkhauser, 1992.

[8] Meyer P. A. Quantum Probability for Probabilists // Lecture Notes in Math. V. 1338. Berlin: Springer-Verlag, 1993.

[9] Lindblad G. On the generators of quantum dynamical semigroups // Comm. Math. Phys. 1976. V. 48. № 2. P. 119-130.

[10] Gorini V., Kossakovsky A., Sudarshan E. C. G. Completely positive dynamical semigroups of $n$-level systems // J. Math. Phys. 1976. V. 17. № 3. P. 821-825.

[11] Березин Ф. А. Метод вторичного квантования. М.: Наука, 1986.

[12] Чеботарев А. М. О симметричной форме стохастического уравнения Хадсона-Партасарати // Матем. заметки. 1996. Т. 60. № 5. С. 726-750.

[13] Fagnola F. On quantum stochastic differential equations with unbounded coefficients // Prob. Theory and Related Fields. 1990. V. 86. P. 501-516.

[14] Fagnola F. Unitarity of solutions to quantum stochastic differential equations and conservativity of the associated semigroup // Quantum Probability and Related Topics. V. VII. Singapore: World Scientific, 1992. P. 139-148.

[15] Fagnola F. Characterization of isometric and unitary weakly differentiable cocycles in Fock space // Quantum Probability and Related Topics. V. VIII. Singapore: World Scientific, 1993. P. 143-164.

Московский государственный университет

Поступило

им. М. В. Ломоносова

13.12.2002

E-mail: alex@chebotar.phys.msu.su 